\title{
OBSERVATION AND SIMULATION OF THE NONLINEAR DEPENDENCE OF VACUUM PRESSURES ON THE POSITRON BEAM CURRENT AT THE KEKB
}

\author{
Y. Suetsugu, KEK, Tsukuba, Japan.
}

\begin{abstract}
A non-linear dependence of vacuum pressure on the beam current has been observed in the KEKB positron ring. The behaviour changes with the bunch fill pattern, the bunch current and the external magnetic field, which is similar to that of the beam size blow up phenomenon. A simple simulation on the non-linear pressure rise is proposed assuming that the behaviour occurs by the electron multipactoring. The simulation well reproduces the observed non-linear pressure behaviours. The results support a suggestion that the beam size blow up is caused by the electron cloud around the positron beam.
\end{abstract}

\section{INTRODUCTION}

The KEK B-factory (KEKB) is an electron-positron collider with asymmetric energies to detect the $\mathrm{CP}$ violation in bottom-quark decay [1]. The KEKB consists of two rings, that is, the High Energy Ring (HER) for 8.0 $\mathrm{GeV}$ electrons and the Low Energy Ring (LER) for 3.5

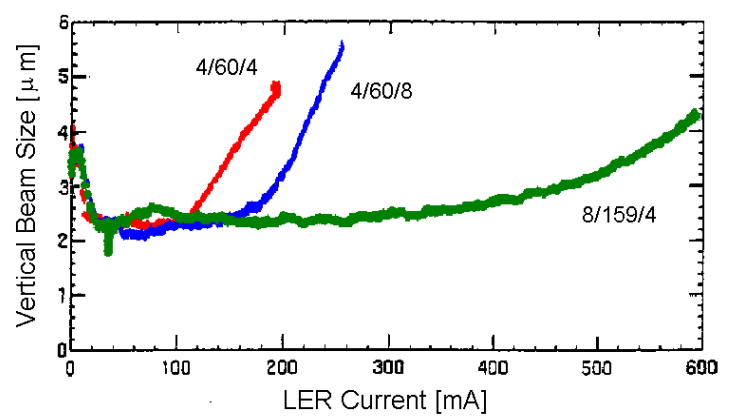

Figure 1: Dependences of vertical beam size on the beam current for three bunch patterns. The symbol of $4 / 60 / 8$, for an example, means that the beam consists of 4 trains of 60 bunches filled with every 8 RF buckets spacing.

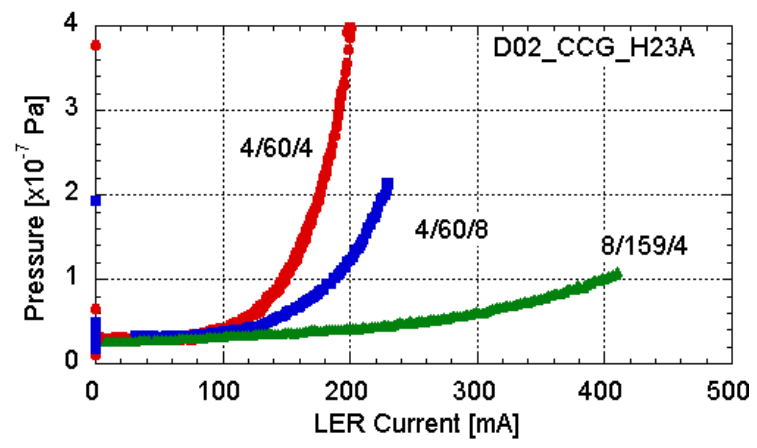

Figure 2: Dependence of the pressure measured at a gauge at a straight section on the beam current for three bunch patterns same as that in Figure 1.
$\mathrm{GeV}$ positrons.

At present, the blow up of the vertical beam size of positron beam is the most important issue for KEKB since it should limit the luminosity. The blow up depends on the bunch fill pattern as shown in Figure 1, for an example. Many simulations have been performed and the blow up is now considered to be due to the electron cloud around the beam [2]. The seed of the electron cloud is mainly the photoelectrons emitted from the chamber surface irradiated by the synchrotron radiation (SR).

On the other hand, the non-linear pressure rise against the beam current has been observed in the LER as shown in Figure 2, for an example. The pressure rise does not seem to be due to only the synchrotron radiation, where the pressure is simply in proportion to the beam current. The behaviour has characteristics as follows:

(1) The non-linearity is strong at the place without magnetic field (the straight section). Actually, the non-linearity reduces by applying a solenoid magnetic field of several gausses.

(2) The pressure increases rapidly at some beam current, that is, shows a resonant phenomenon.

(3) The different bunch patterns and the bunch currents give the different behaviours of pressure rise.

(4) The behaviour is similar to that of the blow up of the vertical beam size (see Figure 1 and 2).

The non-linear pressure rise, therefore, may be due to the multipactoring of the electrons and have some relation to the beam blow up issues.

Here a simple simulation was proposed assuming that the non-linear pressure rise is due to the multipactoring of the electrons [3]. The simulation well reproduced the observed pressure dependences on the bunch fill pattern and so on. The results support the suggestion that the beam size blow up occurs by the electron cloud around the positron beam.

\section{SIMULATION}

\subsection{Model and calculation}

The model for the simulation is presented in Figure 3. The geometry is two dimensions. Consider a bunched positron beam, with a bunch length and a bunch radius of $l_{b}$ and $r_{b}$, running in a vacuum chamber with the light speed. The space between the adjacent bunches is $s_{b}$.

At the beginning, a photoelectron is emitted from the chamber surface. When a bunch is above the electron, it is attracted by the bunch. Otherwise, the electron drifts freely with an initial energy. At an appropriate timing, the electron is accelerated by the succeeding bunches and may hit again the chamber after an average interval of $\tau_{e}$ 
Beam Chamber
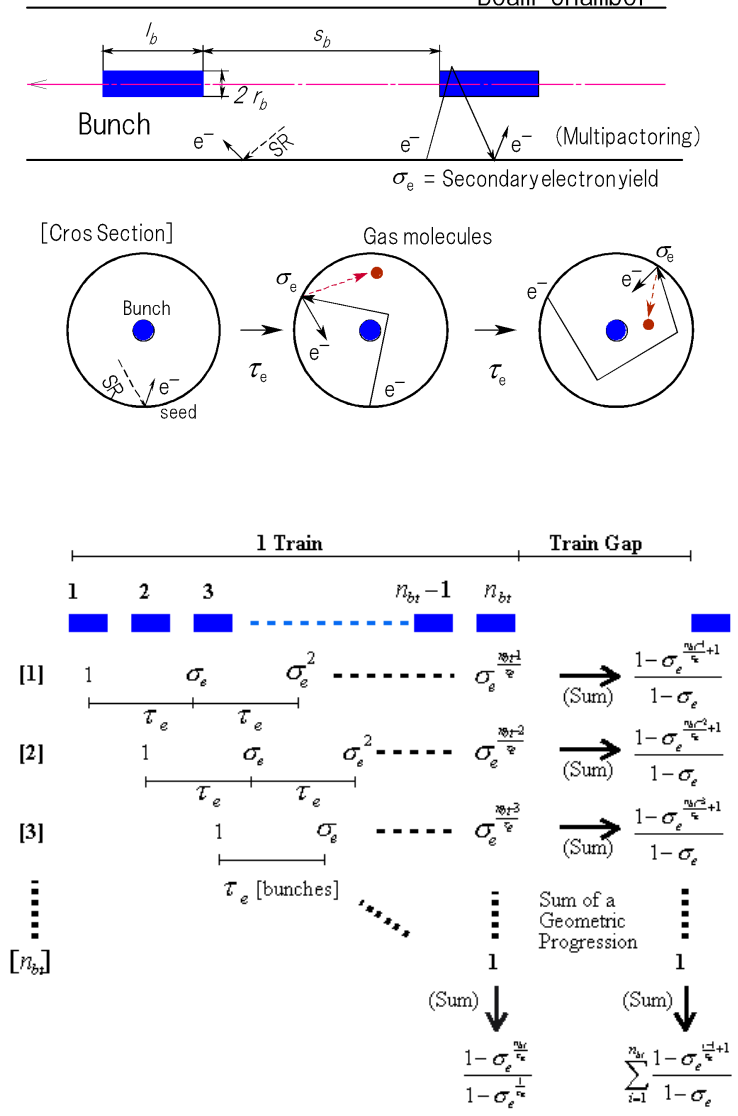

Figure 4: Principle of pressure calculation.

in the unit of bunches. The secondary electrons are generated with an average probability of $\sigma_{e}$, the secondary electron yield. The secondary electrons repeat the same process above (multipactoring). As is well known, the $\sigma_{e}$ depends on the electron energy. In the simulation, therefore, a number of test electrons emitted at various timings with different initial energies are traced following the equation of motion, and the $\sigma_{e}$ and the $\tau_{e}$ are calculated.

The pressure is considered to be determined by the EID (Electron Impact Desorption). The pressure is, therefore, almost proportional to the total number of generated electrons in a train of bunch since the gas molecules would remains in the chamber while the train is passing. Then once $\sigma_{e}$ and the $\tau_{e}$ are obtained, the pressure, $P$, is given by

$$
P \propto I_{\text {bunch }} \times N_{\text {train }} \times \sum_{i=1}^{n_{b t}} \frac{1-\sigma_{e}^{\frac{i-1}{\tau_{e}}-1}}{1-\sigma_{e}},
$$

where $I_{\text {bunch }}$ is the bunch current, which is proportional to the number of photons emitted from a bunch. The $N_{\text {train }}$ is the number of trains and a train consists of a series of $n_{b t}$ bunches. The last term is the number of generated electrons in one train. The term is a sum of a geometric progression as explained in Figure 4. If the $\sigma_{e}$ is 0 , the $P$ is only proportional to the total beam current $\left(=I_{\text {bunch }} \times N_{\text {train }}\right.$ $\left.\mathrm{X} n_{b t}\right)$.

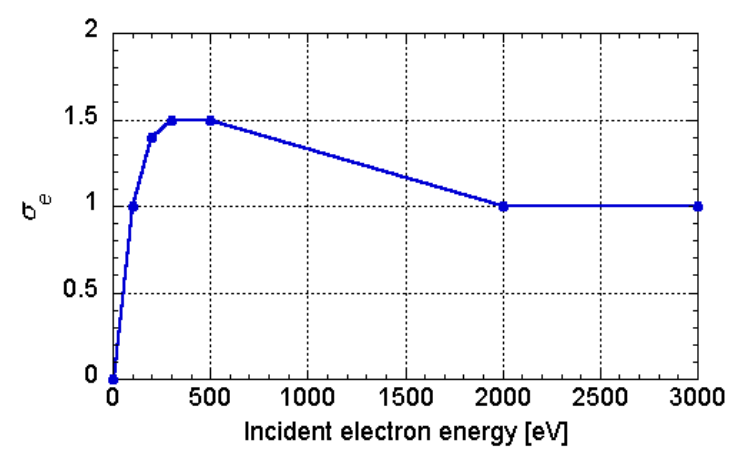

Figure 5: Assumed energy dependence of the secondary yield, $\sigma_{e}$, for a copper surface.

\subsection{Assumption}

In the calculation, the following conditions are assumed.

(1) Initial energy of emitted electrons is in the range $2-8$ $\mathrm{eV}$.

(2) $r_{b}=200 \mu \mathrm{m}, l_{b}=20 \mathrm{~mm}$ (about $3 \sigma_{z}$ ), and $s_{b}=4-12$ RF buckets ( 1 bucket $=2 \mathrm{~ns}$ ).

(3) No axial motion (two dimensions).

(4) No space charge effect.

(5) EID coefficient, i.e., the number of gas molecules emitted for one electron, is constant.

(6) Chamber is a circular pipe with a radius of $47 \mathrm{~mm}$ (same as that for the LER).

(7) Angular distribution of the emitted electrons follows the cosine law.

(8) Tracking starts at a random time between adjoining bunches.

(9) Electrons and gas molecules inside the chamber is completely cleared during a train gap.

(10) Secondary electron yield $\sigma_{e}$ is a function of the incident electron energy as shown in Figure 5 [4].

\section{RESULTS AND DISCUSSIONS}

\subsection{Dependence on bunch fill pattern}

The dependences of the calculated pressures (in arbitrary unit) on the beam current are shown in Figure 6 for several bunch fill patterns. The behaviours against the fill patterns are just similar to that in Figure 2 although the correspondent beam current is somewhat different. The dependence on the fill pattern comes from the change of resonant conditions of the multipactoring. Actually the dependences were different for a vacuum chamber with a different radius. The initial energy of electrons was not so sensitive to the results. Compared for the fill patterns of $4 / 60 / 4$ and $8 / 159 / 4$, it is found that the non-linear pressure rise is also dependent on the bunch current.

The results indicate that the dependence of non-linear pressure rise on the fill pattern can be explained by the electron multipactoring. That means the number of electrons in the chamber also changes with a resonance phenomenon for different fill patterns as the pressure. In the simulation, actually, the number of electrons remained 


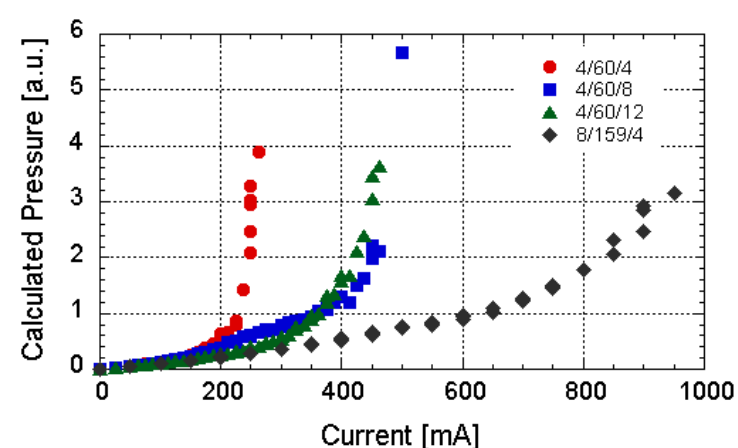

Figure 6: Dependences of calculated pressures on the beam current for 4 different fill pattern.

at the end of a train has a similar structure as Equation (1) (see Figure 4). Remember here that the dependence of pressure on the fill patterns is very similar to that of the beam size blow up as shown in Figure 1. The simulation results, therefore, support the suggestion that the blow up is caused by the electron cloud around the positron beam.

\subsection{Dependence on magnetic field}

Figure 7 shows the behaviours of pressures against the beam current at a gauge, where a solenoid was winded around the beam chamber. Without the magnetic filed, the pressure shows a non-linear behaviour against the beam current $(\mathrm{W} / \mathrm{O})$. As shown in the figure, however, the pressure increases linearly against the beam current with a solenoid field of about $10 \mathrm{G}(\mathrm{W} /)$. Figure 8 is the calculated results for with and without solenoid field. The behaviour is just similar to the actual pressure's one. In the simulation that can be explained by the change of the trajectory of the electrons, that is, the energy of hitting electrons becomes low, i.e., $\sigma_{e} \quad<<1$. Then the multipactoring does not occur. The results again indicate that the non-linear pressure rise is determined by the electron multipactoring.

The effect of the magnetic field strength on the pressure behaviour is also examined changing the solenoid current. The result indicates that the magnetic filed of about $6 \mathrm{G}$ is sufficient to clear the non-linear pressure rise. The simulation also gives the similar result although the behaviour is different for small magnetic field.

Actually it is found that the solenoid field larger than 40 $\mathrm{G}$ is effective to suppress the blow up of the vertical beam size [5]. The results here again support the suggestion that the electron cloud leads to the beam size blow up.

\section{SUMMARY}

The non-linear pressure behaviour has been observed in the KEKB LER (positron ring). The pressure behaviour seems to have some relation to the blow up of the beam size. A simple two dimensional simulation was performed assuming that the pressure behaviour occurs by the electron multipactoring. The results reproduced the observed pressure dependence on the bunch fill pattern

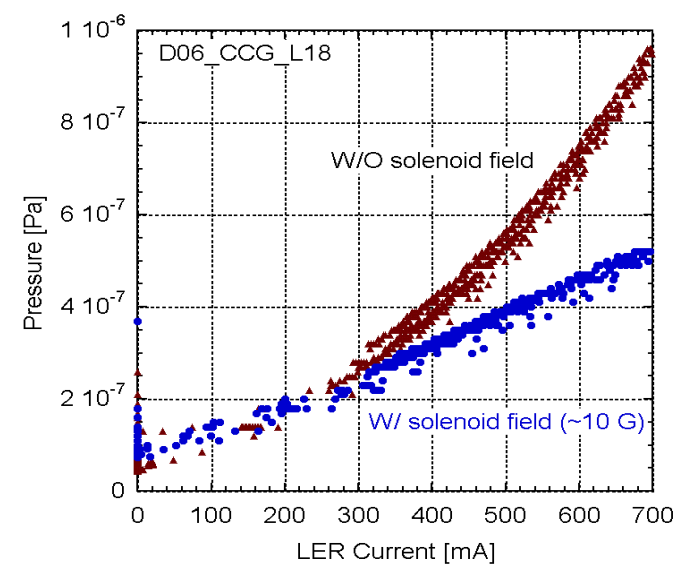

Figure 7: Beam current dependence of pressures for with and without solenoid field of about $10 \mathrm{G}$.

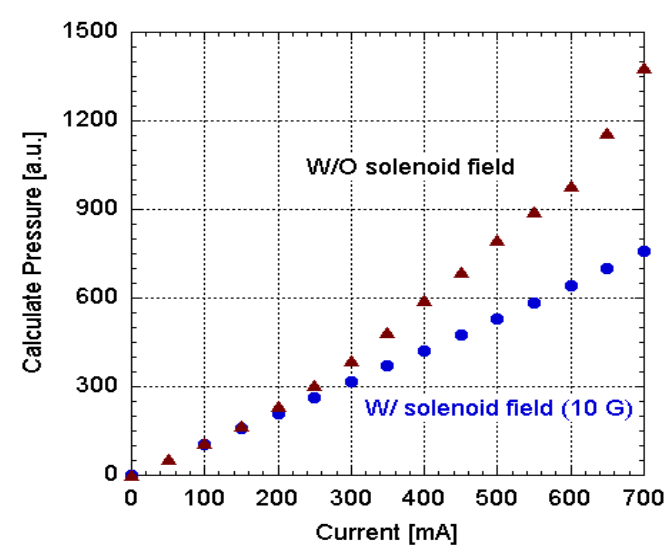

Figure 8: Calculated pressure rise against beam current for with and without solenoid field of $10 \mathrm{G}$.

and the external magnetic field. These support the suggestion that the electron cloud causes the beam size blow up.

Note here that the simulation here is only two dimensions and cannot include the movement of electrons axial direction. Further study is necessary to clarify the non-linear pressure rise in detail and to investigate the relation to the blow up of the positron beam size.

\section{REFERENCES}

[1] S.Kurokawa, "Present Status of KEKB Project", 6th EPAC, Stockholm, June 1998.

[2] H.Fukuma et al., "Observation of vertical beam blowup in KEKB Low Energy Ring", Proceedings of EPAC 2000, Vienna, p.1122.

[3]O.Grobner, "BUNCH

INDUCED MULTIPACTORING", Proceedings of The X HEACC, Protvino, 1977, p.277.

[4] S.Kato, a private communication.

[5] H.Fukuma et al., "STUDY OF VERTICAL BEAM BLOWUP IN KEKB LOW ENERGY RING", Proceedings of HEACC2001, Tsukuba. 